\title{
Editorial
}

\section{Progress in Microwave Imaging: From Theoretical Developments to Cutting-Edge Applications}

\author{
Ibrahim Akduman, ${ }^{1}$ Lorenzo Crocco, ${ }^{2}$ Amélie Litman, ${ }^{3}$ and Ali Yapar ${ }^{1}$ \\ ${ }^{1}$ Istanbul Technical University, Istanbul, Turkey \\ ${ }^{2}$ National Research Council of Italy (CNR-IREA), 80124 Naples, Italy \\ ${ }^{3}$ Aix Marseille Université, CNRS, Centrale Marseille, Institut Fresnel UMR 7249, 13397 Marseille, France
}

Correspondence should be addressed to Ibrahim Akduman; akduman@itu.edu.tr

Received 2 November 2015; Accepted 4 November 2015

Copyright (C) 2015 Ibrahim Akduman et al. This is an open access article distributed under the Creative Commons Attribution License, which permits unrestricted use, distribution, and reproduction in any medium, provided the original work is properly cited.

Microwave imaging is an increasingly important area of applied electromagnetics, which is extremely relevant from both a theoretical and technological point of view. In particular, its significance has constantly increased in the last twenty years, also thanks to the joint exploitation of effective inversion strategies, advanced computational methods, and reliable devices. Such a synergy has enabled the successful progress from simulations to actual application in fields as different as medical diagnostics, structural assessment, and security.

With this special issue, gathering nine papers from scholars in the field, we have meant to provide an updated overview on some recent advances in this technology, in terms of emerging applications and experimental validations, as well as methodological advances.

Starting from applications, this collection confirms that the attention of researchers working on microwave imaging is focused on three very challenging fields.

The first application field is subsurface imaging, which is relevant to ground penetrating radar surveys in geophysics or civil engineering and antipersonnel landmine clearance. In this framework, a significant issue is the roughness of the air soil interface. T. U. Gürbüz and B. Aslanyürek deal with the two-dimensional imaging of a target buried under an unknown rough surface. To this end, they propose a two-step procedure, whose first step provides an estimate of the scenario and the second step is devoted to retrieving the target. Conversely, E. Tetik and I. Akduman address the difficulties of imaging three-dimensional targets buried under a rough surface.

The second topic is the imaging of concealed objects, which is faced in security posting and safety monitoring. Several papers in this special issue deal with this application, discussing approaches to addressing it successfully (T. Jin and A. Yarovoy, P. Millot and L. Casadebaig, and G. Zhao et al.). In particular, T. Jin and A. Yarovoy take advantage of a multiinput multioutput array configuration and a preliminary estimation of the wall's feature to enhance the reconstruction results. Conversely, M. N. Akıncı et al. apply qualitative imaging methods to retrieve the shape of hidden targets, whereas P. Millot and L. Casadebaig and G. Zhao et al. deal with the problem of detecting concealed weapons from ultrawide band radar measurements, which calls for very fast and effective imaging devices and processing techniques in order to ensure quick operations.

The third subject is biomedical monitoring, including breast cancer imaging and other diagnostics problems. This is the emerging application of microwave imaging, wherein such a technology can bring significant improvements thanks to the nonionizing nature of microwaves (which means completely safe and harmless examinations) and the intrinsic low cost and portability. However, biomedical monitoring requires the development of suitably designed devices, since the antennas are hosted in a coupling medium. As such, as done by S. I. Latif et al., the antennas have to be designed taking into account the specific nature of the surrounding 
environment, so as to comply with the requirements of the imaging procedures. Also, proper modeling tools are needed in this application, since biomedical devices are typically embedded into a casing, in order to host the coupling medium and shield them from external interferences. Moreover, accurate, quantitative reconstruction methods are needed to support the clinical decisions. These aspects are addressed in the papers by M. Ambrosanio et al. and by J. De Zaeytijd and A. Franchois, respectively.

The experimental validation of the imaging methods is an important aspect, which is needed to ensure a successful translation of the developed techniques into real world scenarios. Therefore, the fact that several papers in the special issue present experimental validations of existing quantitative and qualitative inversion methods is an important circumstance. In particular, T. Jin and A. Yarovoy validate their novel backpropagation algorithm for through-the-wall imaging, whereas the paper by P. Millot and L. Casadebaig and the one by $\mathrm{G}$. Zhao et al. present experimental results concerned with SAR processing for concealed object imaging. S. I. Latif et al. validate a beamforming algorithm for radar medical imaging by applying it to the measurement done with the antennas they have developed. Finally, M. N. Akıncr et al. provide an experimental assessment of qualitative imaging, showing that these very effective methods can successfully work in realistic conditions.

From the methodological point of view, two emerging paradigms in inverse scattering methods arise from the presented papers.

The first one is concerned with the development of advanced regularization tools. This includes the exploitation of compressive sensing techniques (P. Millot and L. Casadebaig and G. Zhao et al.) that are powerful regularization tools to image spare targets, which is often the case in applications where the target of the imaging procedure occupies only a small portion of the region of interest. A new kind of regularization is the one proposed by J. De Zaeytijd and A. Franchois, in which the projection of the approximated Hessian over cosine basis functions is used as a smart preconditioner to enhance the convergence of iterative solution schemes for quantitative biomedical imaging.

The second paradigm is related to the virtual experiments concept. This is a completely new way to approach microwave imaging, based on the simple observation that inverse scattering problems can be handled by conveniently rearranging the available experiments into new, virtual ones. As an example, this concept enables a linearized quantitative inversion method whose validity, analyzed by L. Di Donato et al. with numerical examples and analytical considerations, is significantly broader than the usual Born approximation, in terms of both contrast magnitude and targets size. Moreover, this kind of approach is also viable for real world scenarios, as shown by M. Ambrosanio et al., where the inversion method is extended to the case of embedded systems, typically exploited in biomedical applications. Notably, the MIMO system exploited by T. Jin and A. Yarovoy for the virtual aperture radar is indeed an example of a device that can support microwave imaging procedures based on the virtual experiments concept.
Seen as a whole, the papers compiled in this special issue cover the above aspects in a quite comprehensive way. Hence, we are confident that we have succeeded in offering our readers and peers an up-to-date overview of microwave imaging techniques and applications.

\section{Acknowledgments}

Finally, we would like to thank all the authors that have contributed with their interesting work to this opus and all the anonymous reviewers that have secured its scientific quality.

Ibrahim Akduman Lorenzo Crocco Amélie Litman Ali Yapar 

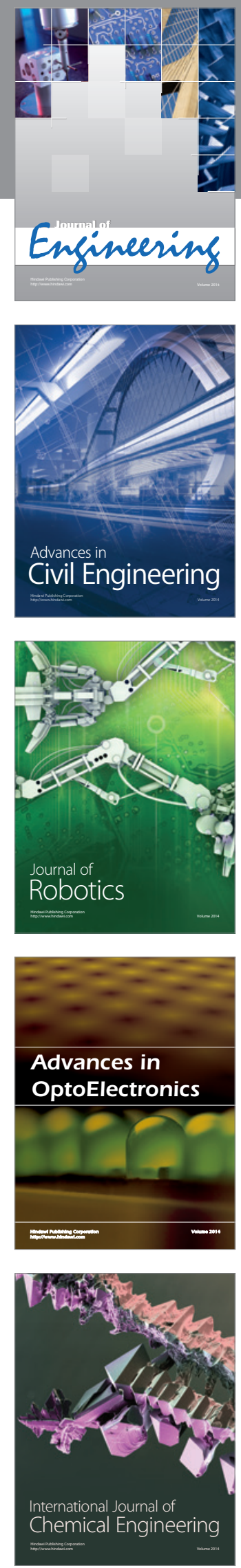

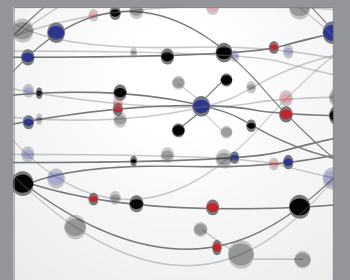

The Scientific World Journal
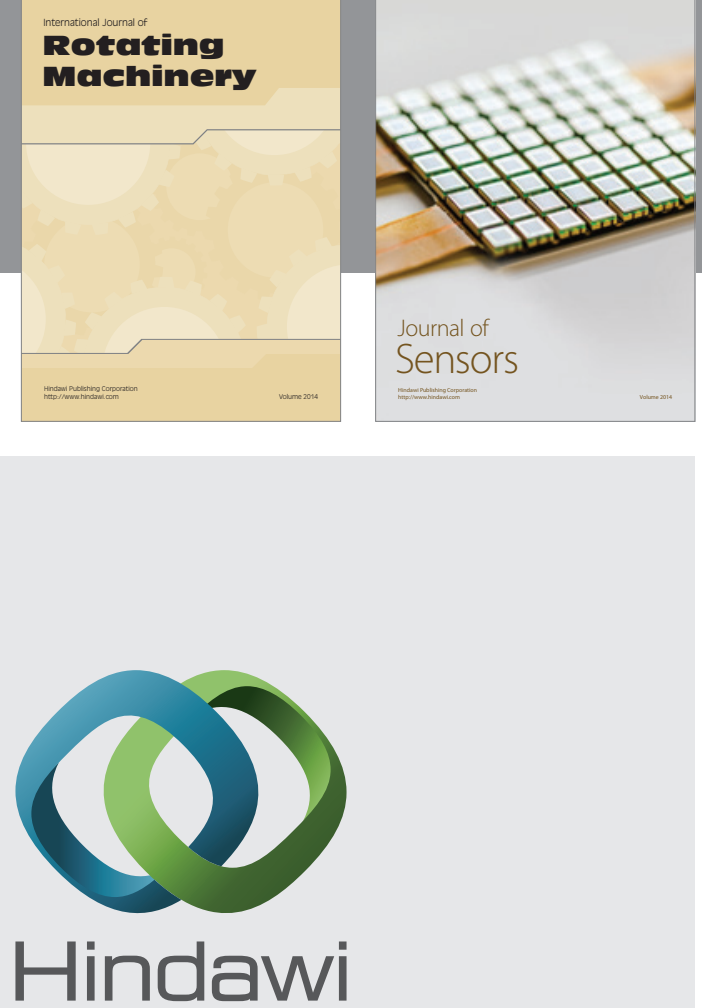

Submit your manuscripts at http://www.hindawi.com
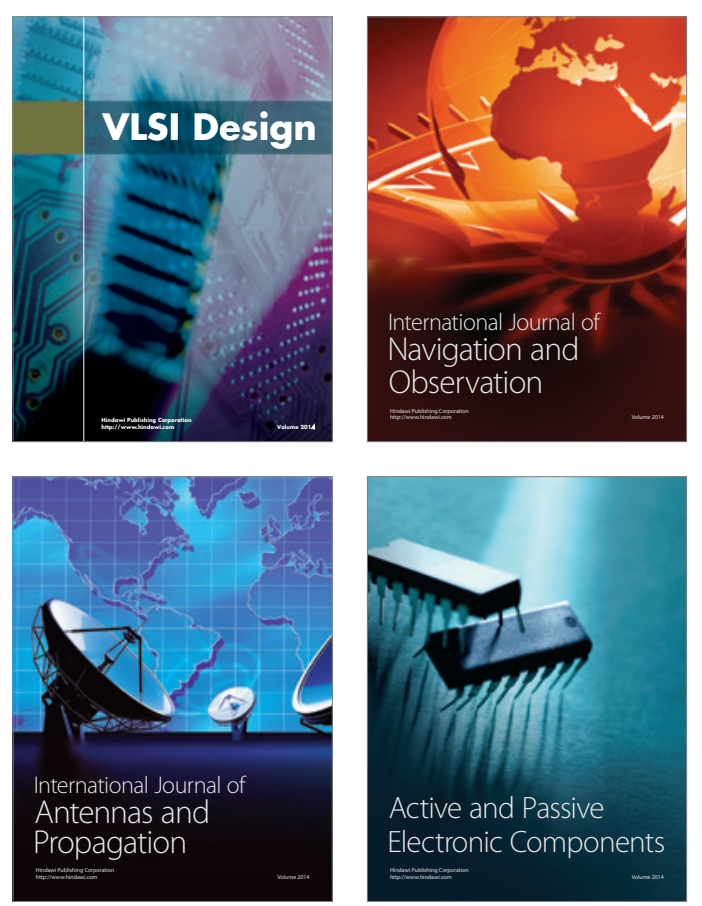
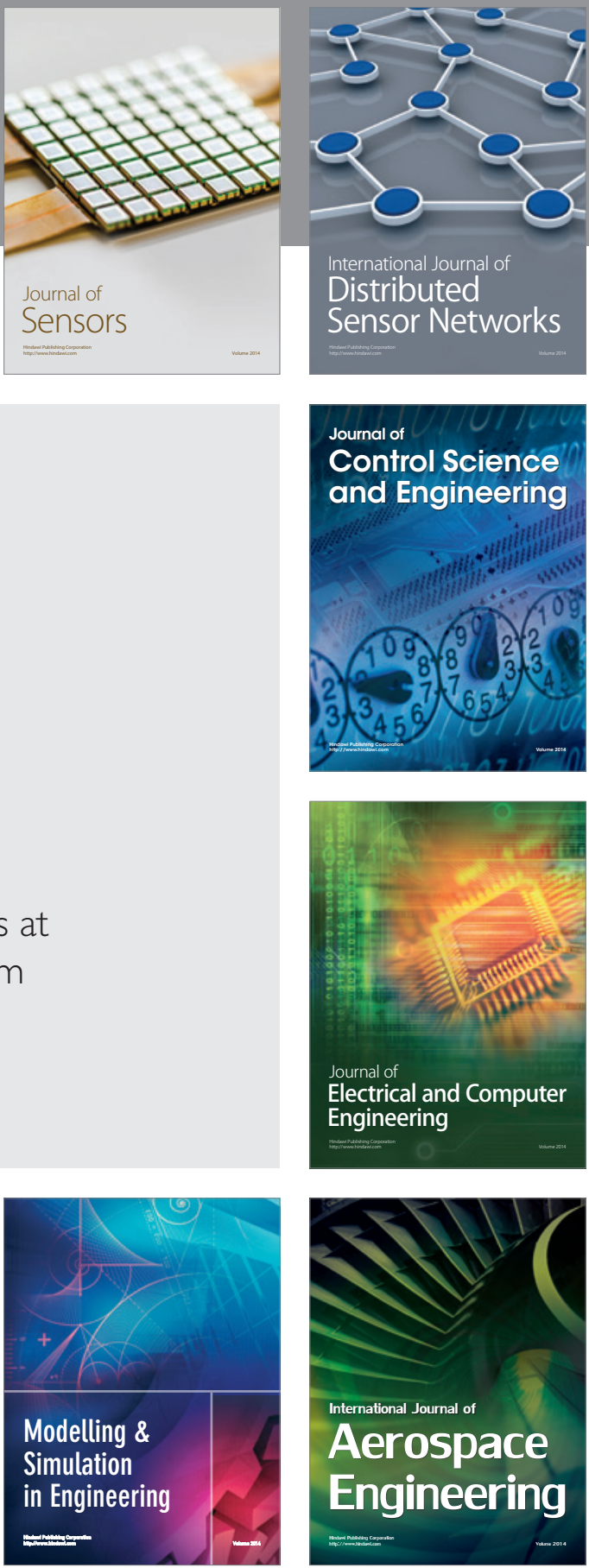

Journal of

Control Science

and Engineering
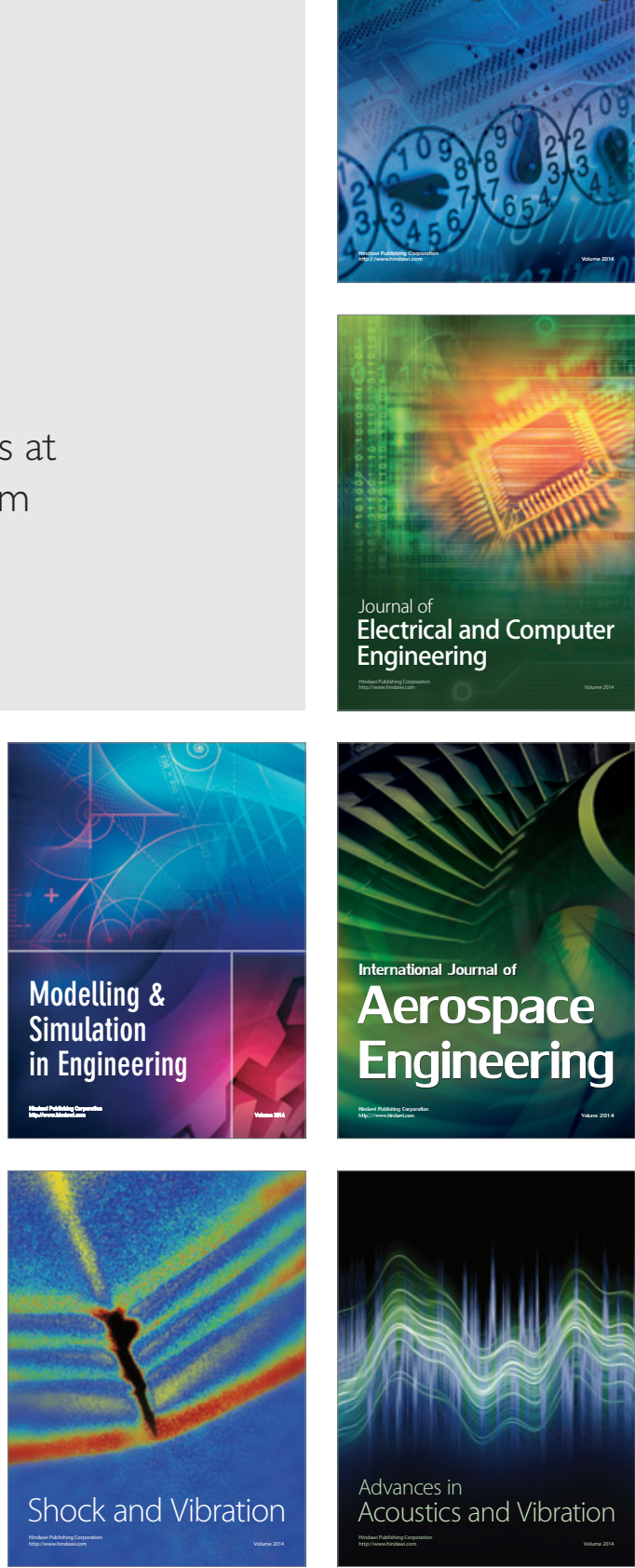